\title{
La exposición a las ondas electromagnéticas en trabajadores de mantenimiento de estaciones de radio base de telefonía celular
}

\section{Exposure to electromagnetic waves in maintenance workers of cell phone base radio stations.}

Enrique Mauricio Barreno Avila. ${ }^{1}$, Pablo Daniel Cardoso Pacheco . ${ }^{2}$, José Geovanny Vega Pérez. ${ }^{3}$ \& Paúl Francisco Freire Ordoñez. ${ }^{4}$

\section{Abstract.}

DOI: $\underline{\text { https://doi.org/10.33262/cienciadigital.v3i4.1155 }}$

The study of the RF fields exposure from cell phone base stations, shows the overexposure to occupational factors of physical risks in this type of tasks. The measurements of power density (s), electric field density (e), magnetic field density $(\mathrm{H})$ performed for heights $(\mathrm{h})$ from 2 to $5 \mathrm{~m}$ in the main lobe of the emitter or antenna where there is maximum gain determined $32 \%$ of the measured points exceed the permissible limit. With these values the safety distances were established, and it was determined that the height is inversely proportional to the electric, magnetic and power field density.

Keywords: Exposure, Electromagnetic Waves, Power Density, Electric Flux Density.

\section{Resumen}

Esta investigación permite dar a conocer los riesgos físicos en las tareas de mantenimiento de una estación de radio base de telefonía celular. A pesar de que hasta la fecha las

1 Faculty of Civil and Mechanical Engineering, Technical University of Ambato, Ambato-Ecuador, enriquebarrenoavila@gmail.com

2 Faculty of Design and Architecture, Technical University of Ambato, Ambato-Ecuador, pablodcardoso@uta.edu.ec

${ }^{3}$ G+ Energy-Risks \& Engineering Group, Department of Food Science and Engineering, Technical University of Ambato, Ambato-Ecuador,jg.vega@uta.edu.ec

4 Academic Faculty Of Sciences and Applied Engineering, Technical University of Cotopaxi, Latacunga-Ecuador, jfrancisco_paul_freire@hotmail.com 
investigaciones realizadas anteriormente no presentan evidencia científica importante para indicar que estas radiaciones afectan al ser humano, estos análisis han determinado la existencia de límites de exposición a radiaciones no ionizantes, los cuales deben ser respetados por los trabajadores que realizan este tipo de trabajos. Las mediciones de densidad de potencia (s), densidad de campo eléctrico (e), densidad de campo magnético $(\mathrm{H})$ realizadas para alturas (h) de 2 hasta $5 \mathrm{~m}$ en el lóbulo principal del emisor o antena donde existe la máxima ganancia determinaron un $32 \%$ de los puntos medidos sobrepasan el límite permisible. Con estos valores se establecieron las distancias de seguridad y, por lo tanto, se determinó que: la altitud es inversamente proporcional a la densidad de campo magnético, eléctrico y de potencia.

Palabras claves: Exposición, Ondas Electromagnéticas, Densidad De Potencia, Densidad De Campo Eléctrico.

\section{Introducción.}

"La generalización del uso de la energía eléctrica y del empleo de radiofrecuencias para la transmisión de la información a distancia, ha dado lugar a una presencia virtualmente ubicua de campos electromagnéticos no ionizantes en el medio ambiente urbano. (Maeso, 2001) durante las últimas décadas, el número de antenas de estaciones base de telefonía móvil que emiten radiación electromagnética (REM) de radiofrecuencia (RF) han aumentado significativamente (Buckus et al., 2017). El uso de esta tecnología aumenta la preocupación sobre los posibles efectos de la REM generada y sus posibles efectos sobre la salud del público en general (Lahham, Alkbash, \& ALMasri, 2016) ya que las RF utilizadas en la comunicación móvil tienen la capacidad de penetrar a través de sustancias semisólidas como la carne y tejido vivo (Mat, D. A. A., 2010)

Las actividades realizadas en ubicaciones cercanas a estaciones base implican la exposición del público a las ondas de radio. (Mann, S., 2006)

La Radio Base (RB) es una parte de todo un conjunto de elementos que componen la red de servicio de telefonía celular y es donde convergen los diferentes riesgos para el trabajador uno de los cuales es el riesgo físico por REM de ondas no ionizantes por su presencia es continua en los procesos de mantenimiento y operaciones (Male, J., 2003).

Los técnicos de mantenimiento en RB de micro-célula y pico-célula en el Ecuador están expuestos de manera continua a las RF a distancias cercanas de la fuente.

"La comisión Internacional para la Protección contra la Radiación No Ionizante (CIPRNI) estableció directrices de actuación basados en los efectos de la salud por los campos electromagnéticos de Radio frecuencia (RF) en el rango de frecuencia de $0 \mathrm{~Hz}$ a $300 \mathrm{GHz}$ " (Protection, 2009) 
Para la medición de la densidad de potencia de las RB de telefonía móvil es necesario el uso de equipos sofisticados y costosos que no siempre están disponibles en los laboratorios y generalmente los datos sobre las antenas suelen ser proveídos por los operadores de telefonía celular. (Mann, S., 2000) Esta investigación evalúa la intensidad de campo eléctrico, la intensidad de campo magnético y la densidad de potencia de la onda plana equivalente, usando un equipo NARADA SRM-3000, para después comparar con los límites permisibles proporcionados por la CIPRNI. Para esto, no se tomo en cuenta los niveles de exposición simultánea por efectos de múltiples fuentes ya que las mediciones fueron realizadas dentro del área de RB que tienen una sola fuente de emisión.

La tabla 1 muestra los límites dispuestos en la normativa ecuatoriana para este tipo de exposición, siendo estos límites; los mismos limites indicados por: "International Committee on Nonionizing Radiation Protection (ICNIRP)”. (FREIRE, 2015)

Tabla No.1: Límite máximo de exposición por estación radio eléctrica fija

\begin{tabular}{|c|c|c|c|c|}
\hline Tipo de exposición & $\begin{array}{l}\text { Rango de } \\
\text { frecuencias }\end{array}$ & $\begin{array}{l}\text { Intensidad de } \\
\text { campo eléctrico, } \mathrm{E} \\
(\mathrm{V} / \mathrm{m})\end{array}$ & $\begin{array}{l}\text { Intensidad de } \\
\text { campo magnético, } \\
\mathrm{H}(\mathrm{A} / \mathrm{m})\end{array}$ & $\begin{array}{l}\text { Densidad de potencia de } \\
\text { onda plana equivalente, } \mathrm{S} \\
(\mathrm{W} / \mathrm{m} 2)\end{array}$ \\
\hline \multirow{6}{*}{ Ocupacional } & $3-65 \mathrm{kHz}$ & 610 & 24,4 & - \\
\hline & $\begin{array}{c}0,065-1 \\
\mathrm{MHz}\end{array}$ & 610 & $1,6 / f$ & - \\
\hline & $1-10 \mathrm{MHz}$ & $610 / f$ & $1,6 / f$ & - \\
\hline & $10-400 \mathrm{MHz}$ & 61 & 0,16 & 10 \\
\hline & $\begin{array}{c}400-2000 \\
\mathrm{MHz}\end{array}$ & $3 \mathrm{f} 1 / 2$ & $0,008 f^{1 / 2}$ & $\mathrm{f} / 40$ \\
\hline & $2-300 \mathrm{GHz}$ & 137 & 0,36 & 50 \\
\hline \multirow{6}{*}{ Poblacional } & $3-150 \mathrm{kHz}$ & 87 & 5 & - \\
\hline & $0,15-1 \mathrm{MHz}$ & 87 & $0,73 / f$ & - \\
\hline & $1-10 \mathrm{MHz}$ & $87 / f^{1 / 2}$ & $0,73 / f$ & - \\
\hline & $10-400 \mathrm{MHz}$ & 28 & 0,073 & 2 \\
\hline & $\begin{array}{c}400-2000 \\
\mathrm{MHz}\end{array}$ & $1,375 f^{1 / 2}$ & $0,0037 \mathrm{f} 1 / 2$ & $\mathrm{f} / 200$ \\
\hline & $2-300 \mathrm{GHz}$ & 61 & 0,16 & 10 \\
\hline
\end{tabular}

Nota. Fuente: Reglamento de Radiaciones no Ionizantes 01-01 Concejo Nacional de Telecomunicaciones 2005. (FREIRE, 2015)Dónde:

- $\quad$ Los valores límites señalados en esta tabla corresponden a valores eficaces (RMS) sin perturbaciones. 
- $\quad f$ corresponde a la magnitud de la frecuencia indicada en la columna rango de frecuencias; se deben omitir las unidades al momento de hacer el cálculo del límite respectivo.

- $\quad$ Para las frecuencias entre $100 \mathrm{kHz}$ y $10 \mathrm{GHz}$, el período de tiempo en el que se debe realizar la medición será de 6 minutos.

Para las frecuencias superiores a $10 \mathrm{GHz}$; el período de tiempo en el que se debe realizar la medición será 68/f1.05 minutos.

En los estudios epidemiológicos realizados a personal expuesto a campos electromagnéticos de radio frecuencias se han tenido varios efectos los cuales se han clasificado en:

- $\quad$ Efectos Térmicos.

- $\quad$ Efectos no Térmicos.

\section{Efectos térmicos}

Estos efectos son los que más estudios presentan, los cuales se definen como el cambio de energía electromagnética a energía térmica. (FREIRE, 2015)

La radiación no ionizante, no tienen la energía suficiente para ionizar la materia, pero si tiene la capacidad de transformar la energía radiante incidente en energía rotacional, la consecuencia de esta transformación es el aumento de la energía cinética molecular lo que conlleva la fricción de molecular y por tanto se produce un efecto térmico.

El efecto genera un incremento en la temperatura; esta puede ser corporal y de forma irregular se distribuye por el interior del organismo, causando que se generen gradientes de temperatura, en determinadas zonas del corporales.

\section{Efectos no térmicos}

Para tratar de explicar estos efectos en radiaciones no ionizantes de radio frecuencia existen diferentes teorías, que se producen afectaciones a órganos internos sin que la temperatura corporal se cambie.

Estas teorías que aún están en investigación son:

- Interacción a nivel molecular, celular o tisular (membranas biológicas).

- Interferencias directas con fenómenos bioeléctricos (alteraciones registradas en el electroencefalograma y electromiograma).

- Alteraciones en la transmisión de la información genética.

La realización de un modelo predictivo, de las consecuencias biológicas de la exposición a radiaciones no ionizantes de radio frecuencia, es impredecible al momento debido a que no se ha estructurado un como la energía es absorbida y distribuida por el cuerpo, se debe notar que la 
irregularidad del cuerpo humano y la particularidad de cada individuo, ha permitido que sea complicado establecer un patrón a seguir.

Las afectaciones que se producen en el cuerpo humano, cuando la temperatura exceden la capacidad de disipación del sistema termorregulador del organismo, tiene como consecuencia una hipertermia, que a su vez desencadena lesiones localizadas, quemaduras, hemorragias, necrosis y muerte tisular.

Cuando la exposición de densidad de potencia es mayor a $10 \mathrm{~mW} / \mathrm{cm}^{2}$, esta será capaz de causar elevación en la temperatura corporal, los órganos que más propensos al daño son los que tiene menor vascularización, como son los ojos y los testículos. (FREIRE, 2015)

\section{Metodología}

\section{Materiales y Equipos}

Según el artículo 9 del reglamento de Radiaciones no Ionizantes 01-01 emitido por el Consejo Nacional de Telecomunicaciones CONATEL en el 2005, actualmente denominado Agencia de Regulación y control de la telecomunicación ARCOTEL, menciona que: "El informe Técnico de Inspección de emisiones de RNI generadas por uso de frecuencias del Espectro Radioeléctrico, será determinado entre otros en base de los siguientes instrumentos": (FREIRE, 2015)

Medidores isotrópicos de radiación. - Son instrumentos isotrópicos para medir campos electromagnéticos y sus componentes, como la intensidad de campo eléctrico, intensidad de campo magnético y la densidad de potencia. Estos equipos tienen la capacidad de considerar el producto de todas las componentes de frecuencia que estén dentro del ancho de banda del instrumento (Barón, Márquez, \& Gómez, 2014). Los Medidores de campo o analizadores de espectro y juego de antenas calibradas para los distintos rangos de medición son instrumentos selectivos, o sintonizables de frecuencia, que permiten conocer el campo electromagnético y sus componentes, en una frecuencia sintonizada o establecida o una banda estrecha de frecuencias (Del Valle, Valdez, Miranda, \& Schlesinger, 2004). Para este estudio se utilizó el equipo NARADA SRM3000 diseñado para actividades de campo y no se usa para realizar mediciones en altas frecuencias en un rango de $100 \mathrm{kHz}$ a $3 \mathrm{GHz}$. (FREIRE, 2015)

\section{Método de Medición}

La metodología de medición se basó en el Reglamento de Radiaciones no Ionizantes 01-01, considerando los siguientes pasos (Flores Arroba, Cayambe, \& Rocio, 2017).

a. Se realizó un levantamiento visual del lugar de instalación del sistema irradiante y se creó una evidencia fotográfica.

b. Se efectuó la medición en los puntos accesibles al público. 
d. Los puntos de medición se encontraron a una distancia no inferior a $20 \mathrm{~cm}$ de cualquier objeto, para evitar posibles efectos de acoplamientos capacitivos.

f. Se calculó el punto de frontera entre el campo cercano y el campo lejano, considerando la ecuación 1 así:

$$
\operatorname{Max}\left(3 \lambda ; \frac{2 D^{2}}{\lambda}\right)
$$

Donde:

D: Diámetro de la antena $(\mathrm{m})$.

$\lambda$ : Longitud de onda (m).

La longitud de onda fue determinada por:

$$
\lambda=\frac{c}{f}
$$

Donde:

c: Velocidad de la luz $(299.792 .458 \mathrm{~m} / \mathrm{s}$ aprox. 3x108 m/s).

f: Frecuencia.

El encargado de la realizar las mediciones debió colocarse en el límite del cálculo teórico de la zona ocupacional y de la zona poblacional, cubriendo un área radial cada 30 grados. Este procedimiento se debió repetir para cada radial (Flores Arroba et al., 2017). Una vez establecidos y cumplidos los límites máximos de exposición se procedió con levantamiento de la señalización visible al público en general y a los operarios en el caso de la zona de rebasamiento. Se siguió las instrucciones del anexo3 del Reglamento de RNI 01-01 CONATEL 2005.

\section{Determinación de la distancia de seguridad}

a) A partir del peor de los casos se estableció la densidad de potencia de la onda plana equivalente con la ecuación 3 , teniendo en consideración el campo lejano.

$$
S_{\text {lim }}=P I R E / \pi R^{2}
$$

Donde:

Slim: Densidad de Potencia de la onda plana equivalente $\left(\mathrm{W} / \mathrm{m}^{2}\right)$.

PIRE: Producto de la potencia suministrada a la antena y la máxima ganancia respecto a una antena isotrópica, y sin tomar en cuenta las pérdidas (W).

$\mathrm{R}$ : Es la distancia entre el punto central de la fuente radiante y el supuesto individuo expuesto a Campos Electro-Magnéticos (m).

b) Se calculó la distancia entre el punto central de la fuente radiante y el supuesto individuo expuesto a campo electromagnético con 1 ecuación 4.

$$
R^{2}=X^{2}+(h-d)^{2}
$$

Donde:

$\mathrm{X}$ : Distancia del individuo al emisor. 
d: Altura del individuo.

h: Para definir el límite máximo de la zona de exposición poblacional y ocupacional se consideró los valores de Slím definidos en la determinación de la distancia de seguridad.

Para la ubicación de la señalización para la Zona Poblacional o de conformidad y Zona Ocupacional se observó las recomendaciones del Reglamento de Radiaciones no Ionizantes 0101 CONATEL 2005, anexo 7 y la recomendación K.52, debiendo debió estar visible tanto al público como al operario de la instalación de estaciones radioeléctricas fijas.

Se demarcó la zona de acuerdo a la topografía del área donde se implantaron vallas que permitían el acceso únicamente al operario, de acuerdo con lo que establece la Superintendencia de Comunicaciones (SUPERCOM).

\section{Resultados}

Resultado de las mediciones de radiaciones no ionizantes.

Las mediciones realizadas en el lóbulo principal de la antena (máxima ganancia), a $2 \mathrm{~m}$ de altura de la antena (h) se muestran en la tabla 2.

Tabla 2. Medición de radiación no ionizante en radio base a $2 \mathrm{~m}$ de altura del lóbulo principal de la antena.

\begin{tabular}{cccccc}
\hline Punto de medición & $\begin{array}{c}\mathbf{h} \\
(\mathbf{m})\end{array}$ & $\begin{array}{c}\mathbf{X} \\
(\mathbf{m})\end{array}$ & $\begin{array}{c}\mathbf{s} \\
\left(\mathbf{m W} / \mathbf{c m}^{2}\right)\end{array}$ & $\mathbf{e}(\mathbf{V} / \mathbf{m})$ & $\begin{array}{c}\mathbf{H} \\
(\mathbf{A} / \mathbf{m})\end{array}$ \\
\hline Pto. 1 & 2 & 0,25 & 5119,39 & 1237,83 & 63,92 \\
Pto.2 & 2 & 0,5 & 3199,62 & 773,64 & 39,95 \\
Pto. 3 & 2 & 1 & 1279,85 & 309,46 & 15,98 \\
Pto. 4 & 2 & 1,5 & 639,92 & 154,73 & 7,99 \\
Pto. 5 & 2 & 2 & 376,43 & 91,02 & 4,7 \\
Pto. 6 & 2 & 2,5 & 246,12 & 59,51 & 3,07 \\
Pto. 7 & 2 & 3 & 172,95 & 41,82 & 2,16 \\
Pto. 8 & 2 & 3,5 & 127,98 & 30,95 & 1,6 \\
Pto. 9 & 2 & 4 & 98,45 & 23,8 & 1,23 \\
Pto. 10 & 2 & 4,5 & 78,04 & 18,87 & 0,97 \\
Pto. 11 & 2 & 5 & 63,36 & 15,32 & 0,79 \\
\hline
\end{tabular}

Nota: las abreviaturas manejadas en la tabla corresponden de la siguiente manera: altura (h), distancia (x), densidad de potencia (s), densidad de campo eléctrico (e), densidad de campo magnético $(\mathrm{H})$. La ganancia máxima de la antena $=17,4(\mathrm{dBi})$; Altura de la medición=1,5 m; Valor del Pire=502,34 W; Potencia máxima equivalente a la salida de la antena $=43,1 \mathrm{~W}$; Limite permisible de exposición (ICNIRP) densidad de potencia Slim =212,5 (mW/cm2); Limite permisible 
de exposición (ICNIRP) densidad de campo eléctrico = 51,38 V/m; Limite permisible de exposición (ICNIRP) densidad de campo magnético $=2,65 \mathrm{~A} / \mathrm{m}$.

En la tabla 2. se observa que el $45 \%$ de los puntos medidos sobrepasan el límite permisible y el 55\% está dentro de los límites permisibles de exposición a radiaciones no ionizantes.

Así mismo para trabajos de este tipo la distancia segura sería a 2,5 m de distancia horizontal respecto al lóbulo principal de la antena.

Las mediciones realizadas en el lóbulo principal de la antena (máxima ganancia), a $3 \mathrm{~m}$ de altura de la antena (h) se muestran en la tabla 3.

Tabla 3. Medición de radiación no ionizante en radio base a $3 \mathrm{~m}$ de altura del lóbulo principal de la antena.

\begin{tabular}{cccccc}
\hline Punto de medición & $\mathbf{h}(\mathbf{m})$ & $\mathbf{x}(\mathbf{m})$ & $\begin{array}{c}\mathbf{s} \\
\left(\mathbf{m W} / \mathbf{c m}^{\mathbf{2}}\right)\end{array}$ & $\mathbf{e}(\mathbf{V} / \mathbf{m})$ & $\mathbf{H}(\mathbf{A} / \mathbf{m})$ \\
\hline Pto. 12 & 3 & 0,25 & 691,81 & 167,27 & 8,64 \\
Pto. 13 & 3 & 0,5 & 639,92 & 154,73 & 7,99 \\
Pto. 14 & 3 & 1 & 492,25 & 119,02 & 6,15 \\
Pto. 15 & 3 & 1,5 & 355,51 & 85,96 & 4,44 \\
Pto. 16 & 3 & 2 & 255,97 & 61,89 & 3,2 \\
Pto. 17 & 3 & 2,5 & 188,21 & 45,51 & 2,35 \\
Pto. 18 & 3 & 3 & 142,21 & 34,38 & 1,78 \\
Pto. 19 & 3 & 3,5 & 110,33 & 26,68 & 1,38 \\
Pto. 20 & 3 & 4 & 87,66 & 21,2 & 1,09 \\
Pto. 21 & 3 & 4,5 & 71,1 & 17,19 & 0,89 \\
Pto. 22 & $\mathbf{3}$ & $\mathbf{5}$ & $\mathbf{5 8 , 7 1}$ & $\mathbf{1 4 , 2}$ & $\mathbf{0 , 7 3}$ \\
\hline
\end{tabular}

Nota: las abreviaturas manejadas en la tabla corresponden de la siguiente manera: altura (h), distancia (x), densidad de potencia (s), densidad de campo eléctrico (e), densidad de campo magnético $(\mathrm{H})$. La ganancia máxima de la antena $=17,4(\mathrm{dBi})$; Altura de la medición=1,5 m; Valor del Pire=502,34 W; Potencia máxima equivalente a la salida de la antena $=43,1 \mathrm{~W}$; Limite permisible de exposición (ICNIRP) densidad de potencia Slim = 212,5 $(\mathrm{mW} / \mathrm{cm} 2)$; Limite permisible de exposición (ICNIRP) densidad de campo eléctrico $=51,38 \mathrm{~V} / \mathrm{m}$; Limite permisible de exposición (ICNIRP) densidad de campo magnético = 2,65 A/m.

En la tabla 3. se observa que el 55\% de los puntos medidos sobrepasan el límite permisible y el $45 \%$ está dentro de los límites permisibles de exposición a radiaciones no ionizantes.

Así mismo para trabajos de este tipo la distancia segura sería a $2 \mathrm{~m}$ de distancia horizontal respecto al lóbulo principal de la antena. 
Las mediciones realizadas en el lóbulo principal de la antena (máxima ganancia), a $4 \mathrm{~m}$ de altura de la antena (h) se muestran en la tabla 4.

Tabla 4. Medición de radiación no ionizante en radio base a $4 \mathrm{~m}$ de altura del lóbulo principal de la antena.

\begin{tabular}{cccccc}
\hline Punto de medición & $\mathbf{h}(\mathbf{m})$ & $\mathbf{x}(\mathbf{m})$ & $\begin{array}{c}\mathbf{s} \\
\left(\mathbf{m W} / \mathbf{c m}^{2}\right)\end{array}$ & $\mathbf{e}(\mathbf{V} / \mathbf{m})$ & $\mathbf{H}(\mathbf{A} / \mathbf{m})$ \\
\hline Pto. 23 & 4 & 0,25 & 253,44 & 61,28 & 3,16 \\
Pto. 24 & 4 & 0,5 & 246,12 & 59,51 & 3,07 \\
Pto. 25 & 4 & 1 & 220,66 & 53,35 & 2,76 \\
Pto. 26 & 4 & 1,5 & 188,21 & 45,51 & 2,35 \\
Pto. 27 & 4 & 2 & 156,08 & 37,74 & 1,95 \\
Pto. 28 & 4 & 2,5 & 127,98 & 30,95 & 1,6 \\
Pto. 29 & 4 & 3 & 104,91 & 25,37 & 1,31 \\
Pto. 30 & 4 & 3,5 & 86,48 & 20,91 & 1,08 \\
Pto. 31 & 4 & 4 & 71,9 & 17,39 & 0,9 \\
Pto. 32 & $\mathbf{4}$ & $\mathbf{4 , 5}$ & $\mathbf{6 0 , 3 7}$ & $\mathbf{1 4 , 6}$ & $\mathbf{0 , 7 5}$ \\
Pto. 33 & 4 & 5 & 51,19 & 12,38 & 0,64 \\
\hline
\end{tabular}

Nota: las abreviaturas manejadas en la tabla corresponden de la siguiente manera: altura (h), distancia (x), densidad de potencia (s), densidad de campo eléctrico (e), densidad de campo magnético $(\mathrm{H})$. La ganancia máxima de la antena $=17,4(\mathrm{dBi})$; Altura de la medición=1,5 m; Valor del Pire $=502,34 \mathrm{~W}$; Potencia máxima equivalente a la salida de la antena $=43,1 \mathrm{~W}$; Limite permisible de exposición (ICNIRP) densidad de potencia Slim = 212,5 (mW/cm2); Limite permisible de exposición (ICNIRP) densidad de campo eléctrico $=51,38 \mathrm{~V} / \mathrm{m}$; Limite permisible de exposición (ICNIRP) densidad de campo magnético = 2,65 A/m.

En la tabla 4. se observa que el $94 \%$ de los puntos medidos sobrepasan el límite permisible y el $6 \%$ está dentro de los límites permisibles de exposición a radiaciones no ionizantes. Así mismo para trabajos de este tipo la distancia segura sería a $1 \mathrm{~m}$ de distancia horizontal respecto al lóbulo principal de la antena.

Las mediciones realizadas en el lóbulo principal de la antena (máxima ganancia), a $5 \mathrm{~m}$ de altura de la antena (h) se muestran en la tabla 5.

Tabla 5. Medición de radiación no ionizante en radio base a $5 \mathrm{~m}$ de altura del lóbulo principal de la antena.

\begin{tabular}{cccccc}
\hline Punto de medición & $\mathbf{h}(\mathbf{m})$ & $\mathbf{x}(\mathbf{m})$ & $\begin{array}{c}\mathbf{s} \\
\left(\mathbf{m W} / \mathbf{c m}^{2}\right)\end{array}$ & $\mathbf{e}(\mathbf{V} / \mathbf{m})$ & $\mathbf{H}(\mathbf{A} / \mathbf{m})$ \\
\hline Pto. 34 & 5 & 0,25 & 129,93 & 31,42 & 1,62 \\
Pto. 35 & 5 & 0,5 & 127,98 & 30,95 & 1,6 \\
Pto. 36 & 5 & 1 & 120,74 & 29,19 & 1,51 \\
Pto. 37 & 5 & 1,5 & 110,33 & 26,68 & 1,38 \\
Pto. 38 & 5 & 2 & 98,45 & 23,8 & 1,23
\end{tabular}




$\begin{array}{lccccc}\text { Pto. 39 } & 5 & 2,5 & 86,48 & 20,91 & 1,08 \\ \text { Pto. 40 } & 5 & 3 & 75,29 & 18,2 & 0,94 \\ \text { Pto. 41 } & 5 & 3,5 & 65,3 & 15,79 & 0,82 \\ \text { Pto. 42 } & \mathbf{5} & \mathbf{4} & \mathbf{5 6 , 6 3} & \mathbf{1 3 , 6 9} & \mathbf{0 , 7 1} \\ \text { Pto. 43 } & 5 & 4,5 & 49,22 & 11,9 & 0,61 \\ \text { Pto. 44 } & \mathbf{5} & \mathbf{5} & \mathbf{4 2 , 9 5} & \mathbf{1 0 , 3 8} & \mathbf{0 , 5 4}\end{array}$

Nota: Altura (h), distancia (x), densidad de potencia (s), densidad de campo eléctrico (e), densidad de campo magnético $(\mathrm{H})$. La ganancia máxima de la antena $=17,4(\mathrm{dBi})$; Altura de la medición $=1,5 \mathrm{~m}$; Valor del Pire=502,34 W; Potencia máxima equivalente a la salida de la antena $=43,1 \mathrm{~W}$; Limite permisible de exposición (ICNIRP) densidad de potencia Slim $=212,5$ $(\mathrm{mW} / \mathrm{cm} 2)$; Limite permisible de exposición (ICNIRP) densidad de campo eléctrico $=51,38 \mathrm{~V} / \mathrm{m}$; Limite permisible de exposición (ICNIRP) densidad de campo magnético = 2,65 A/m.

En la tabla 5. se puede observar que el $100 \%$ de los puntos medidos están dentro de los límites permisibles de exposición a radiaciones no ionizantes. Así mismo para trabajos de este tipo la distancia segura sería a cualquier distancia horizontal respecto al lóbulo principal de la antena.

\section{Conclusiones.}

- Por los valores obtenidos se concluye que los trabajos en distancias menores a 2 metros de altura desde la base de la antena sobrepasan los límites permisibles determinados por la CIPRNI por lo que se deben prohibir para evitar responsabilidad patronal y evitar alguna enfermedad profesional relacionada. El 68\% sobrepasa el límite exposición profesional para radiaciones no ionizantes.

- Se determinó que la atura es inversamente proporcional a la densidad de campo eléctrico, magnético y de potencia, es decir a mayor altura menor serán las densidades mencionadas y también sucedió lo mismo con las distancias horizontales a mayor distancia horizontal las densidades van disminuyendo en su valor.

- Debido a las mediciones realizadas se identificaron las zonas de rebasamiento del límite permisible de exposición, dentro de una radio base lugar donde se desarrollan las actividades de mantenimiento de sistemas y equipos celulares, determinando así lugares específicos donde la tarea de mantenimiento presenta posibles afectaciones a la salud del trabajador.

- La población laboral en un $61 \%$ cree, que el trabajo bajo la influencia de riesgo físico de los campos electromagnéticos, para radiaciones no ionizantes en las frecuencias de telefonía celular, afecta el clima laboral, y en un $71 \%$ cree que este riesgo afecta su salud física y mental debido al desconocimiento de sus efectos.

\section{Referencias Bibliográficas}

Álvarez Garcia, V. (1999). La Normalización industrial. Valencia: Universidad de Valencia, 1999, 263 p. ISBN 8480028076.

Aponte, G., Escobar, A., Pinedo, C. R., \& Arizabaleta, G. (2007). Medición de campos electromagnéticos en la ciudad de Cali, Colombia. Información tecnológica, 18(3), 39-47.

Avendaño Aguilera, J. V., Gonzaga Orellana, K. A., \& Ruiz Reyes, J. F. (2013). Análisis técnico de las radiaciones electromagnéticas emitidas por las antenas de las radiobases celulares en la ciudad de Guayaquil. 
ALVAREZ HEREDIA, F. Salud Ocupacional. Colombia: Ediciones Ecoc, 2011, 360p.ISB 9789586484701.

Barón, F. E. T., Márquez, C. A. L., \& Gómez, E. S. (2014). Metodología para hacer mediciones de radiación electromagnética de telefonía celular. Revista de Tecnología, 13(2), 67-76.

Brandan, M. E., Perches, R. D., \& Ostrosky, P. (1998). La radiación al servicio de la vida: Fondo de Cultura Económica.

Buckus, R., Strukčinskienė, B., Raistenskis, J., Stukas, R., Šidlauskienė, A., Čerkauskienė, R., . . . Cretescu, I. (2017). A Technical Approach to the Evaluation of Radiofrequency Radiation Emissions from Mobile Telephony Base Stations. International journal of environmental research and public health, 14(3), 244.

Castillo Heredia, L. J. (2014). Estudio de los niveles de radiación electromagnética no ionizante producidas por las antenas de radio, televisión y estaciones base de telefonía celular en varias zonas de la ciudad de Riobamba.

Castillo, L. (2013). Estudio de los niveles de radiación electromagnética no ionizantes producidas por las antenas de radio, televisión y estaciones base de telefonía celular en varias zonas de la ciudad de Riobamba. Tesis de pregrado). Escuela Superior Politécnica de Chimborazo

Cevallos Escobar, M. V. (2000). Tutorial explicativo e interactivo del funcionamiento de un sistema celular. Quito: EPN, 2000.

Cooper, T., Mann, S., Khalid, M., \& Blackwell, R. (2006). Public exposure to radio waves near GSM microcell and picocell base stations. Journal of Radiological Protection, 26(2), 199.

Del Valle, E., Valdez, A., Miranda, C., \& Schlesinger, P. (2004). Contaminación del medio ambiente Medida de Radiaciones No ionizantes de una emisora de FM en la banda de 88 a 108 Mhz, utilizando la norma No 117/03 de la CNC. Argentina: Universidad Nacional del Nordeste.

Freire, p. (2015). Riesgos Físicos en los Campos Electromagnéticos, Radiaciones No Ionizantes y su Incidencia en la Población Laboral del Departamento de Operaciones y mantenimiento de la empresa Guaguitel S.A. Propuesta De Un Sistema De Trabajo Seguro. Latacunga: UTC.

Flores Arroba, A. M., Cayambe, C., \& Rocio, D. (2017). Estudio de las radiaciones electromagnéticas para determinar el patrón de radiaciones de una antena genérica y su comportamiento de radiación. Escuela Superior Politécnica de Chimborazo.

Lahham, A., Alkbash, J. A., \& ALMasri, H. (2016). Theoretical Evaluation of Electromagnetic Emissions from GSM900 Mobile Telephony Base Stations in the West Bank and Gaza Strip-Palestine. Radiation protection dosimetry, 174(1), 74-78. 
Maeso, A. Ú. (2001). Bases biológicas para normativas de protección ante radiaciones no ionizantes: España.

Male, J., d'Inzeo, G., Engstrom, S., Feychting, M., Joyner, K., Kheifets, L., . . Pilla, A. (2003). Niels Kuster Dariusz Leszczynski James Lin Richard Luben. Paper presented at the Abstracts for the Bioelectromagnetics Society Annual Meeting.

Mann, S., Cooper, T., Allen, S., Blackwell, R., \& Lowe, A. (2000). Exposure to radio waves near mobile phone base stations. Radiological Protection Bulletin, 13-16.

Mat, D. A. A., Tat, F. K. W., Kipli, K., Joseph, A., Lias, K., \& Marzuki, A. S. W. (2010). Visualization and analytical measurement of electromagnetic radiation from handheld mobile phones. Paper presented at the 2010 Second International Conference on Computer Engineering and Applications.

Muñoz, V.(2009) Prevención de riesgo - Implantación de un sistema efectivo de control de riesgo. Argentina: Editorial El Cid Editor | apuntes, 14 p.

Pereyra, V. R. (2004). INTRODUCCIÓN A LAS CÁMARAS ANECOICAS PARA MEDICIONES DE CAMPO ELECTROMAGNÉTICO. Paper presented at the Simposio de Metrología. México.

Pérez Alejo, J. L., \& Miranda Leyva, R. (2010). Radiaciones electromagnéticas y salud en la investigación médica. Revista Cubana de Medicina Militar, 39(1), 0-0.

Protection, I. C. o. N.-I. R. (2009). others (ICNIRP) statement on the guidelines for limiting exposure to time-varying electric, magnetic, and electromagnetic fields (up to $300 \mathrm{GHz}$ ),". Health physics, 97(3), 257-258.

Romo Morales, J. A., \& Rubio Mora, D. A. (2011). Impacto ambiental de la radiación no ionizante generada por sistemas de telefonía móvil en el Distrito Metropolitano de Quito. QUITO/EPN/2011.

SUPERTEL (2011). Cobertura y calidad de servicios. Ecuador: Super Intendencia de Telecomunicaciones, $28 \mathrm{p}$.

Tomasi, W. (2003). Sistemas de comunicaciones electrónicas: Pearson educación.

Vargas Marcos, F. (2005). La contaminación ambiental como factor determinante de la salud: SciELO Public Health.

\section{【 Ciencia}




\section{PARA CITAR EL ARTÍCULO INDEXADO.}

Barreno Avila, E. M., Cardoso Pacheco , P. D., Vega Pérez, J. G., \& Freire Ordoñez, P. F. (2019). La exposición a las ondas electromagnéticas en trabajadores de mantenimiento de estaciones de radio base de telefonía celular. Ciencia Digital, 3(4), 250-262. https://doi.org/10.33262/cienciadigital.v3i4.1155

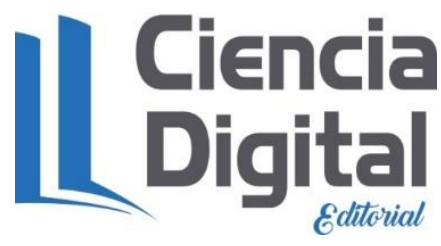

El artículo que se publica es de exclusiva responsabilidad de los autores y no necesariamente reflejan el pensamiento de la Revista Ciencia Digital.

El artículo queda en propiedad de la revista y, por tanto, su publicación parcial y/o total en otro medio tiene que ser autorizado por el director de la Revista Ciencia Digital.
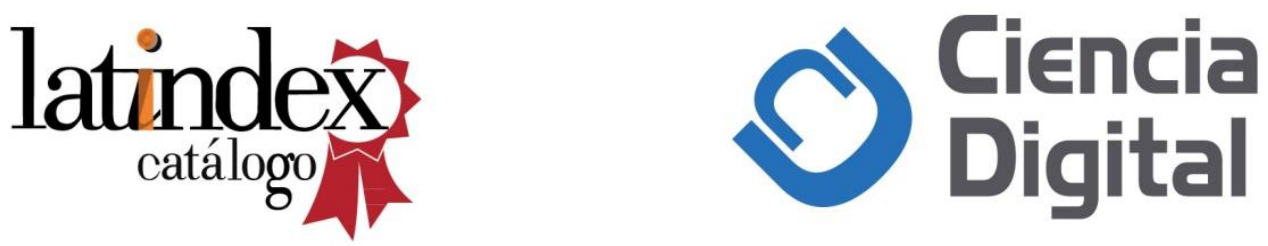